\title{
Poxvirus vectors: orphaned and underappreciated
}

\author{
Michael J. Mastrangelo, ${ }^{1}$ Laurence C. Eisenlohr, ${ }^{2}$ \\ Leonard Gomella, ${ }^{3}$ and Edmund C. Lattime ${ }^{4}$
}
Perspective
SERIES
on cancer biotherapy
David H. Kirn, Editor

${ }^{1}$ Department of Medicine,

${ }^{2}$ Department of Microbiology and Immunology, and

${ }^{3}$ Department of Urology, Thomas Jefferson University, Philadelphia, Pennsylvania, USA

${ }^{4}$ Department of Surgery, University of Medicine and Dentistry of New Jersey University (UMDNJ)-

Robert Wood Johnson Medical School and The Cancer Institute of New Jersey, New Brunswick, New Jersey, USA

Address correspondence to: Edmund C. Lattime, UMDNJ-Robert Wood Johnson Medical School and

The Cancer Institute of New Jersey, 195 Little Albany Street, New Brunswick, New Jersey 08901, USA.

Phone: (732) 235-8588; Fax: (732) 235-8098; E-mail: LattimEC@umdnj.edu.

When one considers the choice of viral vectors for use in gene therapy systems, candidates usually include adenovirus and retrovirus families and, in the case of retrovirus, most recently the HIV-derived lentiviral vectors. These vectors have been chosen based on criteria such as tropism, duration of expression, and capacity to integrate in the host genome. A number of characteristics of their life cycle make poxviruses poor candidates for long-term expression, and, as a result, they are neglected by investigators and rarely represented in reviews and at conferences. However, vaccinia and its relatives may be ideal in immunotherapy applications, including their use as replicating agents that can be directed against solid tumors. Poxvirus vectors, which enable us, as immunologists, to revisit 1 of our greatest triumphs (Figure 1), can be used both to deliver recombinant vaccines and to effect in situ gene transfer to provide cytokines that promote the recognition and rejection of tumors.

\section{Vaccinia virus vectors}

Vaccinia virus and other members of the poxviridae are unusual in that they remain in the cytoplasm and use virally encoded polymerases to carry out replication and transcription. Thus, recombination of viral DNA into the genome is not of concern with vaccinia, as it is with other vectors, particularly retroviruses. The infectious cycle is divided into 3 phases. Early-phase genes, typically encoding proteins with enzymatic function, are expressed before replication. The expression of a small number of intermediate genes depends on replication of the genome and, in turn, drives expression of structural proteins and other products of the late genes (reviewed in ref. 1). Generally speaking, "late" vaccinia promoters drive gene expression more strongly than "early" vaccinia promoters, making late or early/late promoters attractive for controlling expression of transgenes.

Vaccinia virus was the first widely used vaccine and resulted in the eradication of smallpox (variola; Figure 1). As such, vaccinia has the longest and most extensive history of use in humans. Over time, it has been recognized as a relatively safe agent, with infrequent serious side effects, although it does induce a vigorous immune response and can be lethal for those who are immunocompromised or have eczema (1). Although smallpox is highly contagious, transmission being mainly via the respiratory tract, vaccinia is much less so and can be easily confined under standard Biosafety
Level 2 practices. Today, as vaccination of the general population against smallpox has been discontinued, vaccinia is best known as a vector for transient expression of proteins. A clear advantage of vaccinia in this regard is its wide tropism. With variable efficiency, vaccinia infects most cell lines of mammalian origin. Its large genome $(\sim 200 \mathrm{~kb})$ allows the stable insertion of fragments of DNA as large as $25 \mathrm{~kb}(2)$, well above the range of many other vectors.

Generating a vaccinia recombinant is relatively straightforward. The gene of interest is inserted into a recombination plasmid containing a vaccinia virus promoter and 2 segments of the vaccinia virus genome, which flank the pro-

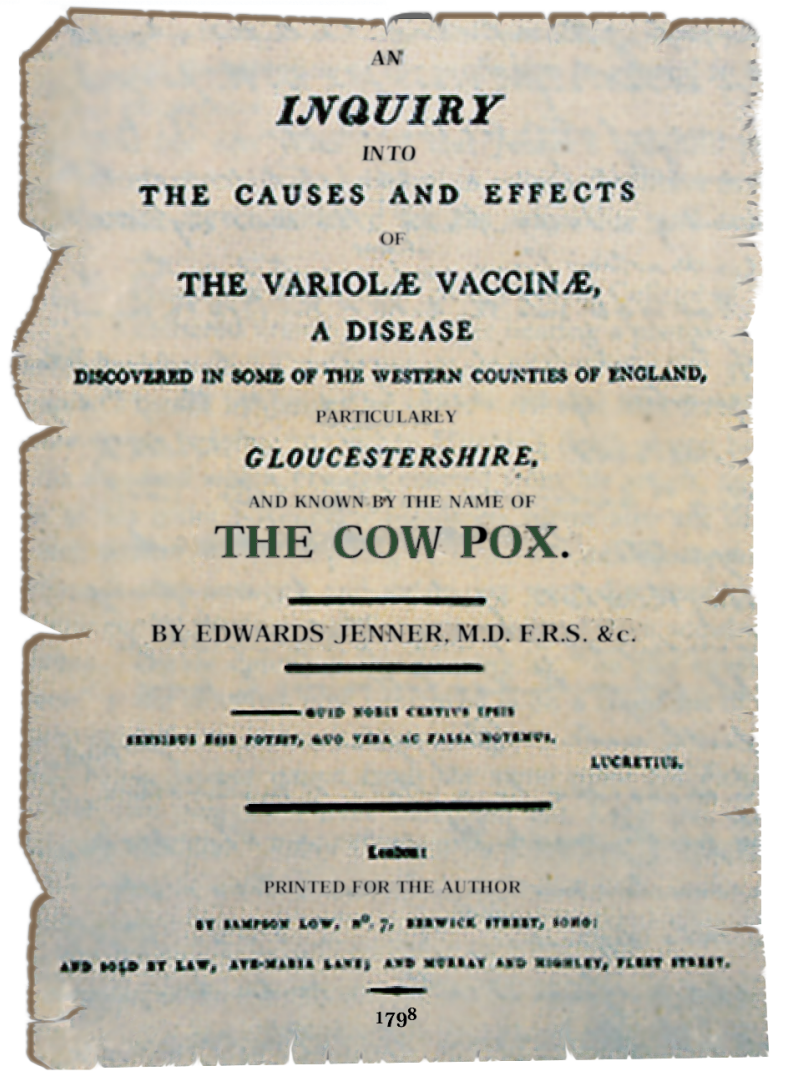

Figure 1

Jenner's 1798 monograph describing the beneficial effects of exposure to variola represents one of immunology's earliest and greatest triumphs. Suitably modified, recombinant viruses now offer the prospect of safe and selective destruction of tumors. 


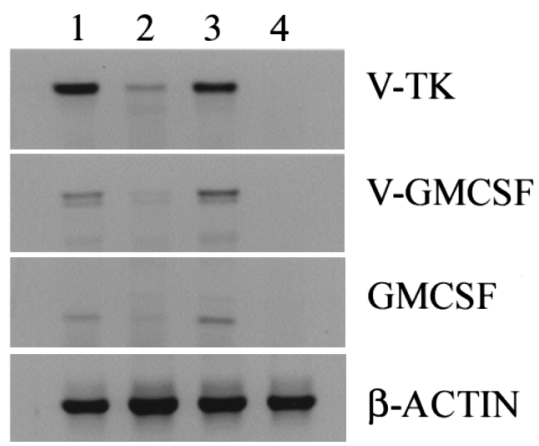

\section{Figure 2}

Persistent expression of vaccinia-encoded transgenes in biopsies of treated melanomas. To determine whether immune responses to the virus would blunt the effect of later doses, we performed RT-PCR on mRNA from biopsies taken from a single patient 18 hours after the last of a series of multiple injections (lane 1 ) or 18 hours after the initial injection of recombinant virus (2 lesions, shown in lanes 2 and 3 ). V-TK and human $\mathrm{V}$-GMCSF were both expressed in injected lesions, early and late in the treatment. Endogenous human GM-CSF was also induced in the injected, but not an uninjected control, lesion.

moter and inserted gene and direct site-specific recombination. The promoter-usually from the viral gene P7.5, which is active during early and late phases of infectDepartment of ion-serves to drive expression of the inserted gene. The most popular site of recombination is the viral thymidine kinase (TK) gene, which is disrupted by the recombination event. Recombination is achieved by infecting cells (often CV-1 cells, derived from the African green monkey), with wild-type vaccinia stock, and then transfecting the infected cells with the recombination plasmid. Recombinant virus will be present in the resultant vaccinia stock at modest frequency, on the order of $0.1 \%$, but can be selected and plaque purified in subsequent passages. The vaccinia recombinant system allows considerable control of heterologous gene expression; modified promoter sequences can drive expression at levels ranging from barely detectable to extraordinarily high (3). Finally, with sufficient care, heterologous DNA, also as large as $25 \mathrm{~kb}$ in length, can be lig- ated directly into the vaccinia genome, obviating the need for recombination and the associated procedures $(1,4)$.

Wild-type vaccinia infection is invariably cytolytic and, as already discussed here, can be lethal in immunocompromised individuals, but less-virulent poxvirus vectors, such as Modified Vaccinia Ankara (MVA) (5) or fowlpox virus, are now available that replicate in avian cells and can infect, but not complete, replication in most mammalian cells (6). Owing to their lack of immunological cross reactivity with vaccinia, fowlpox and other variants confer enhanced vaccine activity when combined with vaccinia. Replication can also be inhibited by treating purified wild-type vaccinia virions with psoralen and UV light, or by engineering viral strains lacking non-essential genes that contribute to virulence.

Vaccinia, and poxviruses in general, are clearly vectors of great utility, and continuing advancements will undoubtedly broaden their applicability. Nevertheless, there are some applications for which they will probably never be suited. Vaccinia expresses on the order of 100 different proteins, at least some of which stimulate a vigorous immune response, as anyone receiving the smallpox vaccine can attest. Thus, the concern arises that expression of the recombinant gene may be limited in individuals with recent exposure to the virus who have developed neutralizing antibody to the vector. In our experience, however, revaccinated subjects, who were vaccinated 25-60 years before therapy, show little or no residual immunity from their first exposure to the virus. Moreover, as discussed later here, transgenes are expressed successfully even after multiple inoculations with the same recombinant poxvirus. We speculate that vaccinia-specific antibodies interfere with release of newly assembled virions from the cell but do not perturb the attachment and entry phase.

\section{Recombinant poxvirus-based vaccines}

Although not traditionally thought of as such, vaccination with recombinant viruses induces the expression of foreign gene products in vivo and satisfies a broad definition of gene therapy. Because they are highly immunogenic and readily engineered, poxvirus recombinants have

Table 1

Intratumoral injection of the vaccinia/GM-CSF recombinant virus in patients with malignant melanoma

\begin{tabular}{|c|c|c|c|c|c|c|c|c|}
\hline Patient & Age/sex & Metastases & $\begin{array}{l}\text { Prior } \\
\text { treatment }\end{array}$ & $\begin{array}{c}\text { Total } \\
\text { lesions/sessions }\end{array}$ & $\begin{array}{l}\text { Total dose } \\
\left(\times 10^{7} \mathrm{PFU}\right)\end{array}$ & $\begin{array}{l}\text { Antivaccinia } \\
\text { titer }\end{array}$ & $\begin{array}{c}\text { Anti- } \beta \text {-galactosidase } \\
\text { titer }\end{array}$ & Response \\
\hline 1 & $81 / \mathrm{F}$ & $\begin{array}{l}\text { Dermal } \\
\text { lymph node }\end{array}$ & Radiation & $12 / 26$ & 74.73 & 180 & 220 & $\begin{array}{l}\text { Partial } \\
\text { None }\end{array}$ \\
\hline 2 & $68 / M$ & $\begin{array}{l}\text { Subcutaneous, } \\
\text { lymph node, lung }\end{array}$ & $\mathrm{BCDT}$, taxol & $12 / 27$ & 82.0 & 80 & 30 & None \\
\hline 3 & $32 / F$ & $\begin{array}{l}\text { Dermal } \\
\text { breast }\end{array}$ & $\begin{array}{c}\text { Radiation, BCDT, } \\
\mathrm{DCV}+\mathrm{IL} 2+\text { IFN, taxol }\end{array}$ & $\begin{array}{c}\text { Dermal } 45 / 56 \\
\text { Breast } 2 / 8\end{array}$ & $\begin{array}{l}207.3 \\
20.5\end{array}$ & 225 & 100 & $\begin{array}{l}\text { Complete } \\
\text { Unknown }\end{array}$ \\
\hline 4 & $61 / F$ & $\begin{array}{l}\text { Dermal, } \\
\text { subcutaneous, } \\
\text { lymph node, lung }\end{array}$ & BCDT, IFN & $9 / 11$ & 49 & 400 & 200 & $\begin{array}{l}\text { Partial } \\
\text { None }\end{array}$ \\
\hline 5 & $71 / \mathrm{F}$ & $\begin{array}{l}\text { Dermal } \\
\text { lymph node }\end{array}$ & $\begin{array}{l}\text { Limb perfusion GP100, } \\
\text { MARTI, IL-12, BCDT }\end{array}$ & $17 / 12$ & 47 & 200 & 75 & $\begin{array}{l}\text { Partial } \\
\text { None }\end{array}$ \\
\hline 6 & $67 / M$ & $\begin{array}{l}\text { Subcutaneous, } \\
\text { lymph node, lung }\end{array}$ & BCDT & $13 / 13$ & 64 & $>300$ & 80 & None \\
\hline 7 & $75 / M$ & Dermal & None & $13 / 11$ & 47.1 & 100 & 50 & Complete \\
\hline
\end{tabular}

BCDT, BCNU + cisplatin + DTIC + tamoxifen; DCV, DTIC + cisplatin + vinblastine. 
been used extensively as vaccines for infectious organisms and, more recently, for tumors. Preclinical studies using a variety of tumor transplants in wild-type and antigenexpressing transgenic mice have provided a strong basis for the use of poxvirus vaccines clinically (reviewed in ref. 7). Nonreplicating vectors, such as avipox or fowlpox, which confer extended expression times and enhanced immunogenicity, are now undergoing clinical trials. Preclinical studies demonstrated that as immunity increases to a particular vector, the vector becomes less suitable to boost a response, but it remains possible to prime with 1 virus and boost with a noncrossreactive second virus. Thus, a current East Coast Oncology Group study aimed at inducing immune responses to the prostate specific antigen (PSA) uses vaccinia-PSA and fowlpox-PSA in combination. More elaborate strategies, combining tumor antigen and immune-enhancing cytokine and/or costimulatory molecules, are currently in preclinical evaluation (7). The already-noted ability to encode upwards of $25 \mathrm{~kb}$ of message in poxvirus vectors provides considerable freedom in devising such combinations (2).

\section{In situ cytokine gene transfection in solid tumors}

After the demonstration in preclinical models that cytokine gene-transfected tumor cell vaccines induced protective, and in some cases therapeutic, responses (8), we turned our attention to introducing immune-active genes into tumors in situ. This approach should allow autologous tumor antigens to be presented in a supportive immunological milieu. Vectors for this purpose require a high efficiency of infection or transfection; a broad host cell tropism, allowing their targeting to multiple tumor types and neighboring tissues; and the capacity to act in repeated applications, even in the presence of increasing antiviral titers. Vaccinia clearly satisfies the first 2 criteria, and, being replicative and lytic, its effects should be amplified considerably over the input dose of virus. As detailed later here (Figure 2), we have found that vaccinia vectors confer high levels of transgene expression even after multiple injections that provoke a strong humoral response to viral antigens.

In our early in vitro studies, we confirmed the wide tropism of vaccinia recombinants, showing that this system allowed high efficiency transfection of multiple murine and human tumor cell lines (9). Subsequently, we demonstrated in a murine model that intravesical vaccinia, expressing the influenza HA protein, infected/transfected bladder carcinoma cells and that preimmunization of mice with vaccinia failed to prevent high efficiency transfection $(10,11)$.

While developing clinical cytokine recombinants, we completed a phase I trial of intratumoral administration of the wild-type vaccinia vector in patients with recurrent superficial melanoma. Here again, we found that locally administered vaccinia could infect tumor cells and that, although antivaccinia antibodies were clearly present, they did not prevent subsequent infection by, or replication of, the vaccinia vector, which continued for at least 4 days after intralesional injection (12).

Next, we generated a clinical grade GM-CSF vaccinia recombinant by encoding the GM-CSF into this same strain of vaccinia virus. The rationale for the initial use of GM-CSF was 2-fold. First, Dranoff et al. (8) had devel-

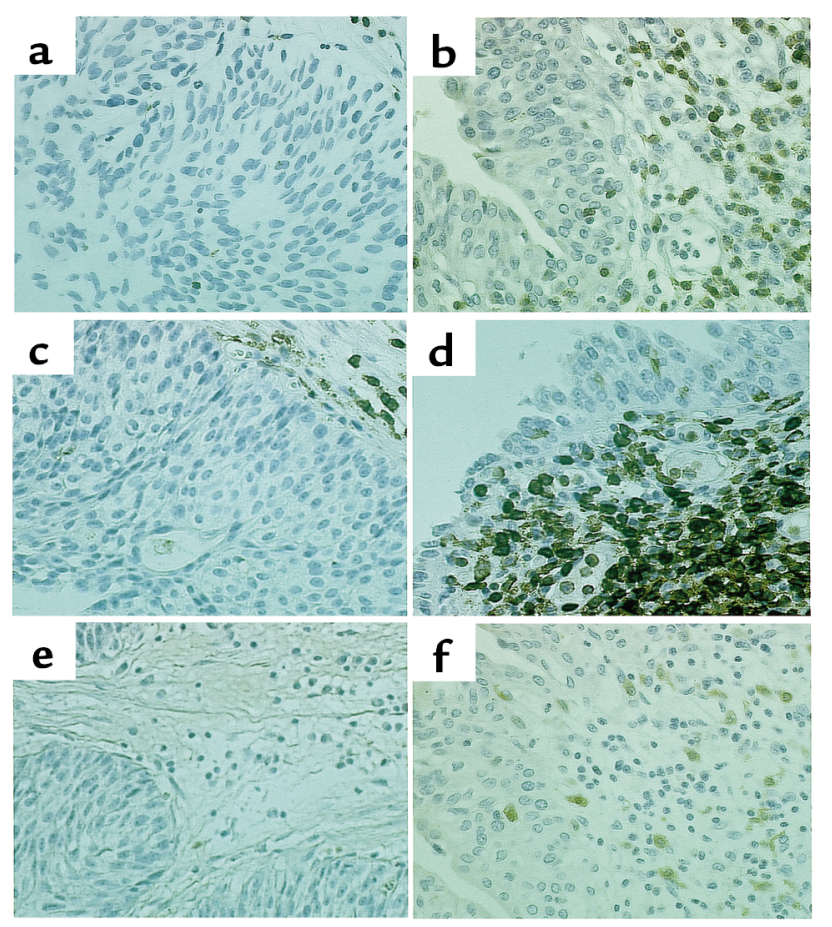

Figure 3

Immunohistochemical staining of bladder from a patient undergoing treatment with vaccinia vector. Pretreatment biopsies (a, $\mathbf{c}$, and $\mathbf{e})$ and post-treatment cystectomy sections ( $\mathbf{b}, \mathbf{d}$, and $\mathbf{f}$ ) were stained for the T-cell marker CD3 ( $\mathbf{a}$ and $\mathbf{b}$ ); the CD45RO marker ( $\mathbf{c}$ and $\mathbf{d}$ ), which is found on activated T cells; and factor XIIla (e and $\mathbf{f}$ ), a dendritic cell marker. The presence of activated $T$ cells and dendritic cells in the tumor infiltrates suggests that the treatment has induced a robust immunologic response.

oped vaccines made from transgenic murine melanoma cells and had demonstrated that cells expressing GMCSF conferred superior antitumor immunity, compared with cells expressing other cytokines. Second, significant clinical experience indicated that GM-CSF was safe and effective when administered secondary to chemotherapy to restore myelopoiesis. In light of this history of clinical use, our demonstration that vaccinia could be administered safely to patients with melanoma seemed to bode well for the speedy approval of clinical trials for the GMCSF recombinant virus. We produced the virus by homologous recombination into the viral TK gene, introducing both the GMCSF gene and the gene for $\beta$-galactosidase, which enabled us to identify infected cells histochemically and immunologically.

\section{Table 2}

Intravesical vaccinia vector before cystectomy in patients with muscleinvasive bladder cancer

\begin{tabular}{lcllc}
\cline { 2 - 4 } Patient & Age/sex & $\begin{array}{l}\text { Dose/treatment } \\
\left(\times 10^{6} \mathrm{PFU}\right)\end{array}$ & Toxicity & $\begin{array}{c}\text { Bladder } \\
\text { inflammation }\end{array}$ \\
1 & $57 / \mathrm{F}$ & $1,5,10$ & Mild dysuria & $\begin{array}{c}\text { Slight } \\
\text { Significant }\end{array}$ \\
3 & $36 / \mathrm{M}$ & $10,25,100$ & Mild dysuria & $\begin{array}{c}\text { Significant } \\
4\end{array}$ \\
& $64 / \mathrm{M}$ & $25,100,100$ & Mild dysuria & Significant \\
\hline
\end{tabular}

Patients received three intravesical instillations of vaccinia vector over a 2-week period with cystectomy performed on the day following the last instillation. 
Having been satisfied that this vaccinia vector, which was produced under good manufacturing practices (GMP) conditions by Dyncorp PRI (Gaithersburg, Maryland, USA), met our requirements of safety and efficacy, we are currently carrying on a phase I trial of intralesional vaccinia-GM-CSF in patients with therapy-refractory recurrent melanoma. All patients have accessible dermal or subcutaneous tumors, and some also have visceral disease. After the demonstration of immune competence, which is important given the replicative nature of the vector, subjects are revaccinated with wild-type virus and then receive intralesional injections of escalating doses of recombinant virus, twice weekly. Table 1 summarizes the results seen in the first 7 patients (described in detail in reference ref. 13). At the highest doses, patients received 2 $\times 10^{7} \mathrm{PFU}$ per lesion, with the injection of multiple lesions resulting in up to $8 \times 10^{7}$ plaque-forming units (PFUs) per session. (For comparison, wild-type vaccinia was used as a smallpox immunization at a scarification dose of $2.5 \times 10^{5}$ PFU.) Patients developed antibodies to both vaccinia and the included $\beta$-galactosidase gene product (Table 1). Crucially, we also confirmed using RT-PCR that vacciniaencoded GM-CSF (V-GMCSF) and thymidine kinase (VTK) mRNAs continued to be expressed at high levels (Figure 2). Although we are limited by our ability to biopsy patient lesions repeatedly, these studies clearly demonstrate that viral replication and GM-CSF mRNA occurred 18 hours after intralesional administration, despite peak antivaccinia antibody levels in the serum. Injected lesions in all patients were heavily infiltrated with $\mathrm{CD}^{+}$and $\mathrm{CD}^{+} \mathrm{T}$ lymphocytes, which were positive for $\mathrm{CD} 3$ and for the CD45 RO activation marker. In addition, tissue macrophages and activated B lymphocytes were observed, as were eosinophils, which were probably induced directly by GM-CSF (13). Although immune infiltrates would not be surprising in lesions injected with the replicating virus, the regression of uninjected lesions not in the primary field was also associated with infiltration by large numbers of T lymphocytes, suggesting that the treatment had induced a systemic antitumor response. We plan to demonstrate this response more rigorously in treating the next cohort of patients.

\section{Intravesical vaccinia vector in patients with bladder cancer}

As a first step to our planned expansion of this strategy to the localized treatment of bladder cancer, we have completed a phase I study of nonrecombinant vaccinia vector in patients with advanced transitional cell carcinoma. Here, too, we used vaccinia in a dose escalation study. Each patient received 3 intravesical doses over a 2 -week period. Given safety concerns, we focused on patients with invasive transitional cell carcinoma scheduled for cystectomy and we timed the dose such that the cystectomy occurred 1 day after the third dose. Table 2 summarizes patient characteristics, doses used, and toxicity. As noted in our prior clinical trials $(12,13)$, patients developed high titers of antivaccinia antibody, although maximal titers were measured after cystectomy, because of the shortened course of therapy. Again, treatment was associated with a significant recruitment of activated $\mathrm{T}$ lymphocytes. Figure 3 demonstrates recruit- ment of activated $\mathrm{CD}^{+} \mathrm{T}$ lymphocytes and dendritic cells, which should enhance antitumor immunity.

\section{Conclusions}

We believe that poxvirus vectors merit a second look for gene therapy applications requiring short-term expression of recombinant proteins. The centuries-old use of vaccinia as a highly immunogenic and effective vaccine has, we believe, led investigators to rule out its efficacy as a biotherapeutic, especially given that immunogenicity has become the bane of most other virus-based gene therapy strategies. Vaccinia's ability to maintain highly efficient transgene expression and its broad tissue tropism make it an attractive alternative, particularly for intralesional delivery, to adenovirus, which has a limited target range and is severely hindered by antibody responses. Our demonstration that large amounts of vaccinia can be administered safely in immune competent individuals should also allay a major concern regarding its use clinically. Furthermore, as discussed earlier here, a series of nonreplicating poxviruses are currently being used in vaccine protocols. If they show a similar high level of transfection efficiency, these vectors may find additional utility in gene transfer studies with reduced risk of replication-associated toxicity.

\section{Acknowledgments}

Studies described were supported by the United States Public Health Service grants CA42908, CA55322, CA69253, and CA74543, and American Cancer Society grants IM-742 and EDT-98.

1. Moss, B. 1996. Genetically engineered poxviruses for recombinant gene expression, vaccination, and safety. Proc. Natl. Acad. Sci. USA. 93:11341-11348

2. Smith, G.L., and Moss, B. 1983. Infectious poxvirus vectors have capacity for at least 25,000 base pairs of foreign DNA. Gene. 25:21-28.

3. Davison, A.J., and Moss, B. 1990. New vaccinia virus recombination plasmids incorporating a synthetic late promoter for high level expression of foreign proteins. Nucleic Acids Res. 18:4285-4286.

4. Merchlinsky, M., and Moss, B. 1992. Introduction of foreign DNA into the vaccinia virus genome by in vitro ligation: recombination-independent selectable cloning vectors. Virology. 190:522-526.

5. Sutter, G., and Moss, B. 1992. Nonreplicating vaccinia vector efficiently expresses recombinant genes. Proc. Natl. Acad. Sci. USA. 89:10847-10851.

6. Paoletti, E. 1996. Application of pox virus vectors to vaccination: an update. Proc. Natl. Acad. Sci. USA. 93:11349-11353.

7. Schlom, J., et al. 1999. Strategies in the development of recombinant vaccines for colon cancer. Semin. Oncol. 26:672-682.

8. Dranoff, G., et al. 1993. Vaccination with irradiated tumor cells engineered to secrete murine granulocyte-macrophage colony stimulating factor stimulates potent, specific, and long lasting anti-tumor immunity. Proc. Natl. Acad. Sci. USA. 90:3539-3543.

9. Lattime, E.C., Lee, S.S., Eisenlohr, L.C., and Mastrangelo, M.J. 1996. Insitu cytokine gene transfection using vaccinia virus vectors. Semin. Oncol. 23:88-100.

10. Lee, S.S., Eisenlohr, L.C., McCue, P.A., Mastrangelo, M.J., and Lattime, E.C. 1994. Intravesical gene therapy: in-vivo gene transfer using vaccinia vectors. Cancer Res. 54:3325-3328.

11. Lattime, E., Eisenlohr, L., Gomella, L., and Mastrangelo, M. 1999. The use of vaccinia virus vectors for immunotherapy via in-situ tumor transfection. In Gene therapy of cancer: translational approaches from preclinical studies to clinical implementation. E. Lattime and S. Gerson, editors. Academic Press. San Diego, CA. 125-137.

12. Mastrangelo, M.J., et al. 1995. A pilot study demonstrating the feasibility of using intratumoral vaccinia injections as a vector for gene transfer. Vaccine Res. 4:55-69

13. Mastrangelo, M.J., et al. 1999. Intratumoral recombinant GMCSF-encoding virus as gene therapy in patients with cutaneous melanoma. Cancer Gene Ther. 6:409-422. 